\title{
INFINITE GAMES VIA COVERING PROPERTIES IN IDEAL TOPOLOGICAL SPACES
}

\author{
A.E. Radwana ${ }^{1 \S}$, Essam El Seidyb ${ }^{2}$, R.B. Esmaeelc ${ }^{3}$ \\ ${ }^{1,2}$ Department of Mathematics \\ Faculty of Science \\ Ain Shams University \\ EGYPT \\ ${ }^{3}$ Department of Mathematics \\ Ibn Al-Haitham College of Education \\ Baghdad University \\ IRAQ
}

\begin{abstract}
First, we propound a comment about the Meneger $(X)$ game. We show that player TWO has a winning strategy always per contra that player ONE. So, we define a new game, say $G(\mathcal{C})$, by using the same data of the Meneger $(X)$ without any winning strategy for both players in general.

In this paper, we are using the concept of ideal topological spaces with its covering properties and $I$-compactness to introduce infinitely long games like: $G(\mathcal{C}, \mathcal{I}), \mathcal{G}_{\mathcal{D}}(\mathcal{C}, \mathcal{I}), \mathcal{G}_{\mathcal{O}}(\mathcal{C}, \mathcal{I})$ and $G\left(C^{*}, \mathcal{C}\right)$. So, we show some results that explain many conditions to make anyone of players have winning strategy. Also, the efficacies of some types of ideals on the strategies for players are studied. Finally, comparisons among player's strategies through the equivalent ideal topological spaces are showed.

These have been important topics of research and have been crucial in the development of game theory especially of topological game theory.
\end{abstract}

AMS Subject Classification: 54C08, 54C10, 54D35, 91A05

Key Words: ideal, $I$-compact, locally finite, $\tau^{*}$-open, Meneger game, selection principle, $G(\mathcal{C}, \mathcal{I}), \mathcal{G}_{\mathcal{D}}(\mathcal{C}, \mathcal{I}), \mathcal{G}_{\mathcal{O}}(\mathcal{C}, \mathcal{I}), \mathcal{G}\left(\mathcal{C}^{*}, \mathcal{C}\right)$ and $G(X, I)$

Received: September 7, 2015

Published: February 4, 2016

${ }^{\S}$ Correspondence author (c) 2016 Academic Publications, Ltd. url: www.acadpubl.eu 


\section{Introduction and Preliminaries}

Throughout the present paper $(X, \tau)$ and $(Y, \sigma)$ denote topological spaces with no separation properties assumed. For a subset A of a topological space $(X, \tau)$, $\operatorname{cl}(A)$ and $\operatorname{int}(A)$ denote the closure and interior of $A$ in $(X, \tau)$ respectively.

The subject of ideals in topological spaces has been studied by Kuratowski [10] and Vaidyanathaswamy [13]. An ideal $I$ on a set $X$ is a nonempty collection of subsets of $X$ which satisfies the conditions: (1) $A \in I$ and $B \subseteq A$ implies $B \in I$, (2) $A \in I$ and $B \in I$ implies $A \cup B \in I$. Given a topological space $(X, \tau)$ with an ideal $I$ on $X$ and $\mathcal{P}(\mathcal{X})$ is the set of all subsets of $X$. A set operator ()$^{*}: \mathcal{P}(\mathcal{X}) \rightarrow \mathcal{P}(\mathcal{X})$ is called a local function [13] of A with respect to $\tau$ and $I$ iff for $A \subseteq X, A^{*}(I, \tau)=\{x \in X: A \cap U \notin I$ for every $U \in \tau(x)\}$ where $\tau(x)=\{U \in \tau: x \in U\}$. A Kuratowski closure operator $c l^{*}()$ [13] for a topology $\tau^{*}(I, \tau)$, called the $*$-topology, finer than $\tau$ is defined by $c l^{*}(A)=$ $A \cup A^{*}(I, \tau)$. When there is no chance for confusion, we will simply write $A^{*}$ for $A^{*}(I, \tau)$ and $\tau^{*}$ for $\tau^{*}(I, \tau)$. If $I$ is an ideal on $X$, then $(X, \tau, I)$ is called an ideal topological space.

In an ideal topological space $(X, \tau, I)$, if $A \subseteq X, i n t^{*}(A)$ denote the interior of $A$ in $\left(X, \tau^{*}\right)$. Closed subsets in $\left(X, \tau^{*}\right)$ are called $\tau^{*}$-closed sets. A subset $A$ of an ideal topological space $(X, \tau, I)$ is $\tau^{*}$-closed [13] if and only if $A^{*} \subseteq A$.

For any ideal topological space $(X, \tau, I)$, the collection $\{V-J: V \in \tau$ and $J \in I\}$ is a basis for $\tau^{*}$. The elements of $\tau^{*}$ are called $\tau^{*}$-open sets. A subset $A$ of an ideal topological space $(X, \tau, I)$ is said to be $\tau^{*}$-dense if $c l^{*}(A)=X$. It is clear that, in a space $(X, \tau, I)$ if $I=\{\varnothing\}$, then $\tau=\tau^{*}[6]$ [9].

Recall that if $(X, \tau, I)$ is an ideal topological space and $A$ is a subset of $X$, then $\left(A, \tau_{A}, I_{A}\right)$ is an ideal topological space where $\tau_{A}$ is the relative topology on $A$ and $I_{A}=\{A \cap J: J \in I\}$.

It is interesting to note that if $I$ is a proper ideal (i.e. $X \notin I$ ), then the collection of the complements of the members of $I$ form a filter on $X$. This is why sometimes ideals are called dual filters.

The following collections form important ideals [9] on a topological space $(X, \tau)$ :

(i) $\{\varnothing\}$ or $I_{\{\varnothing\}}$ : the trivial ideal.

(ii) $\mathcal{P}(\mathcal{X})$ : the improper ideal.

(iii) $\mathcal{F}:\left(\mathcal{I}_{\{}\right)$the ideal of all finite sets.

(iv) $C:\left(I_{c}\right)$ the ideal of all countable subsets of $X$.

(v) $I_{K}$ : the ideal of all relatively compact subsets of $X ; I_{K}=\{A \subseteq X: \operatorname{cl}(A)$ is compact\}.

(iv) $I_{n}$ : the ideal of all nowhere dense subsets of $X ; I_{n}=\{A \subseteq X: \operatorname{int}(\operatorname{cl}(A))=$ 
$\varnothing\}$.

A function $f:(X, \tau, I) \rightarrow(Y, \sigma, J)$ is said to be $*$-continuous with respect to $\tau, I, \sigma$ and $J$ if $f:\left(X, \tau^{*}\right) \rightarrow\left(Y, \sigma^{*}\right)$ is continuous [8].

A subset $A$ of an ideal topological space $(X, \tau, I)$ is said to be $I$-compact [11] if for every cover $\left\{U_{\lambda}: \lambda \in \Lambda\right\}$ of $A$ by open sets of $X$, there exists a finite subset $\Lambda_{0}$ of $\Lambda$ such that $A-\cup\left\{U_{\lambda}: \lambda \in \Lambda_{0}\right\} \in I$. An ideal topological space $(X, \tau, I)$ is said to be $I$-compact if $X$ is $I$-compact as a subset.

A family $\left\{F_{i}: i \in \Lambda\right\}$ of subsets of $X$ is said to have the "finite intersection property", denoted by (f.i.p.), if for every finite subset $\Lambda_{0}$ of $\Lambda$ the intersection $\cap\left\{F_{i}: i \in \Lambda_{0}\right\}$ is non-empty [1].

A family of subsets of a topological space $(X, \tau)$ is said to be locally finite family if every point has a neighborhood which intersects only a finite number of them [2].

Many mathematical notions are defined, or characterized, in terms of selection principles of the following two sorts: Let $S$ be an infinite set and $\mathcal{A}, \mathcal{B}$ both be sets whose members are families of subsets of $\mathrm{S}$. Then $S_{\text {fin }}(\mathcal{A}, \mathcal{B})$ denotes the selection principle. That is to say;

For each sequence $\left(A_{n}: n \in N\right)$ of elements of $\mathcal{A}$ there is a sequence $\left(B_{n}\right.$ : $n \in N)$ of finite sets such that for each $n \in N, B_{n} \subset A_{n}$ and $\cup\left\{B_{n}: n \in N\right\}$ is an element of $\mathcal{B}$.

There is a natural game, denoted $G_{f i n}(\mathcal{A}, \mathcal{B})$, associated with $S_{\text {fin }}(\mathcal{A}, \mathcal{B})$. This game is played as follows: There is an inning per positive integer. In the $n-t h$ inning ONE chooses an $A_{n} \in \mathcal{A}$ and TWO responds with a finite set $B_{n} \subset A_{n}$. A play $A_{1}, B_{1}, \ldots, A_{n}, B_{n}, \ldots$ is won by TWO if $\cup\left\{B_{n}: n \in N\right\}$ is an element of $\mathcal{B}$. Otherwise ONE wins.

$S_{1}(\mathcal{A}, \mathcal{B})$ denotes the selection principle in the following meaning: For each sequence $\left(A_{n}: n \in N\right)$ of elements of $\mathcal{A}$ there is a sequence $\left(b_{n}: n \in N\right)$ such that for each $n \in N, b_{n} \in A_{n}$ and $\left\{b_{n}: n \in N\right\}$ is an element of $\mathcal{B}[3]$.

A topological space $(X, \tau)$ is said to have Menger property if for every sequence $\left(A_{n}: n \in N\right)$ of open covers of $X$ there is a sequence $\left(B_{n}: n \in N\right)$ such that for each $n, B_{n}$ is a finite subset of $A_{n}$, and such that $\left\{B_{n}: n \in N\right\}$ is an open cover of $X[7]$.

Hurewcz discovered a very useful description of spaces which have the Meneger property. This description is given most economically in the language of game - theory. Let $(X, \tau)$ be a topological space. Players ONE and TWO play the following infinitely long game: They play an inning for each positive integer. In the $n-t h$ inning ONE chooses an open cover $A_{n}$ of $X$ and TWO responds by selecting a finite subset $B_{n}$ of $A_{n}$. TWO wins the play $A_{1}, B_{1}, \ldots, A_{n}, B_{n}, \ldots$ of this game if $\left\{B_{n}: n \in N\right\}$ is an open cover of $X$. Otherwise, ONE wins. We 
call this game the Meneger game. It is denoted Meneger (X) [12].

For a set $X$ of real numbers, $X$ has the Meneger property iff ONE does not have a winning strategy in the game Meneger $(X)[12]$.

In what follows we are mostly concerned with the question as to whether either player has a winning strategy in a game. A strategy for one of the players is an algorithm that specifies each move of the player in every possible situation. More precisely, in our work we will study infinitely long game for two players. In the $n-t h$ inning player ONE chooses a set $A_{n}$ (in some restricted collection of sets defined by the "rules" of the game). Player Two responds by choosing a set $B_{n}$, i.e.,

ONE: $A_{1}, A_{2}, \ldots, A_{n}, \ldots$

TWO: $B_{1}, B_{2}, \ldots, B_{n}, \ldots$

A strategy for player ONE is a function $\delta=\left\{\delta_{n}: A^{n-1} \times B^{n-1} \rightarrow A_{n}\right.$ such that $\left.\delta_{n}\left(A_{1}, B_{1}, \ldots, A_{n-1}, B_{n-1}\right)=A_{n}\right\}$. Similarly, one can consider that a strategy for player TWO is a function $T=\left\{T_{n}: A^{n} \times B^{n-1} \rightarrow B_{n}\right.$ such that $\left.T_{n}\left(A_{1}, B_{1}, \ldots, A_{n-1}, B_{n-1}, A_{n}\right)=B_{n}\right\}$.

For any game, say $G$, we denote the fact that player ONE has a winning strategy in the game $G$ by "ONE $\uparrow G$ ". If he does not have a winning strategy we write "ONE $\uparrow G$ ". So we denote that player ONE has a losing strategy in the game $G$ by "ONE $\downarrow G$ ". If he does not have a losing strategy we write "ONE $\downarrow G$ ". The meaning of "TWO $\uparrow G$ ", "TWO $\uparrow G$ ", "TWO $\downarrow G$ " and "TWO $\downarrow G$ " is defined analogously with the same notation used for the game $G$.

Our models of this work are special types of infinitely alternating topological games with perfect information on an infinite set $X$ [4], [5].

\section{Games in Ideal Topological Spaces}

In Meneger $(X)$, we note that player TWO has a winning strategy always per contra that player ONE. As the following proposition shows;

Proposition 2.1. In Meneger (X), player TWO has a winning strategy.

Proof. Let $(X, \tau)$ be a topological space. Players ONE and TWO play infinitely meneger $(X)$. We define a strategy $T$ for TWO in the game Meneger $(X)$. In the first move, ONE will choose an open cover $A_{1}$ for $X$, TWO responds by fix $x_{1} \in X$ and selecting an open neighborhood $B_{1}$ of $x_{1}$ such that $B_{1}=T\left(A_{1}\right)$. In the second move, ONE will choose an open cover $A_{2}$ for $X$, TWO responds by fix $x_{2} \in X-B_{1}$ and selecting an open neighborhood $B_{2}$ of $x_{2}$ such that $B_{2}=T\left(A_{1}, B_{1}, A_{2}\right)$. In the $n-t h$ inning ONE chooses an open cover An of $X$, 
TWO responds by fix $x_{n} \in X-\left(B_{1} \cup B_{2} \cup \cdots \cup B_{n-1}\right)$ and selecting an open neighborhood $B_{n}$ of $x_{n}$ such that $B_{n}=T\left(A_{1}, B_{1}, \ldots, A_{n-1}, B_{n-1}, A_{n}\right)$. Since $X=\cup\left\{B_{n}: n \in N\right\}$. Then $T$ wins for TWO in Meneger $(X)$.

In the following definition we introduce a new game, say $G(C)$, by using the same data of the Meneger $(X)$ with change the winning rule of it.

Definition 2.2. Let $(X, \tau)$ be a topological space and $\mathcal{C}$ a collection of all open covers for $X$. we define the infinitely long game $G(\mathcal{C})$ as follows: They play an inning for each positive integer. In the $n-t h$ inning, ONE chooses $A_{n} \in \mathcal{C}$ and TWO responds by selecting a non-empty finite subset $M_{n}$ of $A_{n}$ such that $B_{n}=\cup\left\{U: U \in M_{n}\right\}$. ONE wins the play $A_{1}, B_{1}, \ldots, A_{n}, B_{n}, \ldots$ of this game if $\left\{B_{n}: n \in N\right\}$ is an open cover of $X$. Otherwise, TWO wins.

Proposition 2.3. If $(X, \tau)$ is a compact topological space, then TWO $\downarrow$ $G(\mathcal{C})$.

In the following, we introduce new games by using the concepts of ideal topological spaces.

Definition 2.4. Let $(X, \tau, I)$ be an ideal topological space and $\mathcal{C}$ a collection of all open covers for $X$. We define the infinitely long game $G(\mathcal{C}, \mathcal{I})$ as follows: They play an inning for each positive integer. In the $n-t h$ inning, ONE chooses $A_{n} \in \mathcal{C}$ and TWO responds by selecting a non-empty finite subset $M_{n}$ of An such that $B_{n}=\cup\left\{U: U \in M_{n}\right\}$. ONE wins the play $A_{1}, B_{1}, \ldots, A_{n}, B_{n}, \ldots$ of this game if $X-\cup\left\{B_{n}: n \in N\right\} \in I$. Otherwise, TWO wins.

Proposition 2.5. Let $(X, \tau, I)$ be an ideal topological space. The following statements are hold.

(i) If $X$ is $I$-compact, then $T W O \downarrow G(\mathcal{C}, \mathcal{I})$.

(ii) If $O N E \uparrow G(\mathcal{C})$, then $O N E \uparrow G(\mathcal{C}, \mathcal{I})$.

(iii) If $I=\{\varnothing\}$, then $O N E \uparrow G(\mathcal{C})$ if and only if $O N E \uparrow G(\mathcal{C}, \mathcal{I})$.

(iv) If $I=\mathcal{P}(\mathcal{X})$, then $T W O \uparrow G(\mathcal{C}, \mathcal{I})$.

Definition 2.6. Let $(X, \tau, I)$ be an ideal topological space and $\mathcal{C}$ a collection of all open covers for $X$. We define the infinitely long game $G_{D}(\mathcal{C}, \mathcal{I})$ (resp. $G_{O}(C, I)$ ) as follows: They play an inning for each positive integer. In the $n$ - th inning, ONE chooses $A_{n} \in \mathcal{C}$ and TWO responds by selecting a non-empty finite subset $M_{n}$ of $A n$ such that $B_{n}=\cup\left\{U: U \in M_{n}\right\}$. ONE wins the play $A_{1}, B_{1}, \ldots, A_{n}, B_{n}, \ldots$ of the game $G_{D}(\mathcal{C}, \mathcal{I})\left(\operatorname{resp} . G_{O}(\mathcal{C}, \mathcal{I})\right)$ if $X-c l\left(\cup\left\{B_{n}: n \in N\right\}\right) \in I$ (resp. $\left.X-\cup\left\{c l\left(B_{n}\right): n \in N\right\} \in I\right)$. Otherwise, TWO wins

Proposition 2.7. Let $(X, \tau, I)$ be an ideal topological space. Then the 
following statements are hold.

(i) $\mathrm{ONE} \uparrow G(\mathcal{C}, \mathcal{I})$ implies $O N E \uparrow G_{O}(\mathcal{C}, \mathcal{I})$.

(ii) $O N E \uparrow G_{O}(\mathcal{C}, \mathcal{I})$ implies $O N E \uparrow G_{D}(\mathcal{C}, \mathcal{I})$.

Proof. It follows from the fact that $\cup\left\{\operatorname{cl}\left(B_{n}\right): n \in \Lambda\right\} \subseteq \operatorname{cl}\left(\cup\left\{B_{n}: n \in \Lambda\right\}\right)$ and by the hereditary property of an ideal.

Proposition 2.8. If $(X, \tau, I)$ is an ideal topological space such that every open subset of $X$ is closed, then the following are equivalents.

(i) ONE has a winning strategy in $G(\mathcal{C}, \mathcal{I})$.

(ii) ONE has a winning strategy in $G_{D}(\mathcal{C}, \mathcal{I})$.

(iii) ONE has a winning strategy in $G_{O}(\mathcal{C}, \mathcal{I})$.

Proposition 2.9. If $(X, \tau, I)$ is an ideal topological space such that every open subset of $X$ is dense, then the following statements are hold.

(i) $T W O$ has no winning strategy in $G_{D}(\mathcal{C}, \mathcal{I})$.

(ii) $T W O$ has no winning strategy in $G_{O}(\mathcal{C}, \mathcal{I})$.

Proposition 2.10. Let $Y$ be a clopen subset of an ideal topological space $(X, \tau, I)$ and $\mathcal{C}_{\mathcal{Y}}$ a collection of all open covers for $Y$ with respect to the relative topology. The following statements are hold;

(i) ONE $\uparrow G\left(\mathcal{C}_{\mathcal{Y}}, \mathcal{I}_{\mathcal{Y}}\right)$ on $Y$ implies $O N E \uparrow G(\mathcal{C}, \mathcal{I})$.

(ii) $O N E \uparrow G_{D}\left(\mathcal{C}_{\mathcal{Y}}, \mathcal{I}_{\mathcal{Y}}\right)$ on $Y$ implies $O N E \uparrow G_{D}(\mathcal{C}, \mathcal{I})$.

(iii) $O N E \uparrow G_{O}\left(\mathcal{C}_{\mathcal{Y}}, \mathcal{I}_{\mathcal{Y}}\right)$ on $Y$ implies $O N E \uparrow G_{O}(\mathcal{C}, \mathcal{I})$.

Proof. (i): Let $\delta^{\prime}$ be a winning strategy for ONE in $G\left(\mathcal{C}_{\mathcal{Y}}, \mathcal{I}_{\mathcal{Y}}\right)$ on $Y$. We define $\delta$ to be a winning strategy for ONE in $G(C, I)$ on $X$. Player ONE looks to the first move $A_{1}^{\prime}=\delta^{\prime}(\varnothing)$ of player ONE in $G\left(C_{Y}, I_{Y}\right)$ on $Y$ and take $A_{1}=\delta(\varnothing)=A_{1}^{\prime} \cup(X-Y)$ to be the first move in $G(C, I)$ on $X$. TWO responds by selecting a non-empty finite subset $M_{1}$ of $A_{1}$ such that $B_{1}=\cup\left\{U: U \in M_{1}\right\}$. Now $M_{1}^{\prime}=\left\{U \cap Y: U \in M_{1}\right\} \subseteq A_{1}^{\prime}$ where $B_{1}^{\prime}=\cup\left\{V: V \in M_{1}^{\prime}\right\}$ is a legitimate move for TWO in $G\left(\mathcal{C}_{\mathcal{Y}}, \mathcal{I}_{\mathcal{Y}}\right)$ on $Y$. Player ONE looks to the second move $A_{2}^{\prime}=\delta^{\prime}\left(A_{1}^{\prime}, B_{1}^{\prime}\right)$ of player ONE in $G\left(\mathcal{C}_{\mathcal{Y}}, \mathcal{I}_{\mathcal{Y}}\right)$ on $Y$ and take $A_{2}=\delta\left(A_{1}, B_{1}\right)=$ $A_{2}^{\prime} \cup(X-Y)$ to be the second move in $G(\mathcal{C}, \mathcal{I})$ on $X$. TWO responds by selecting a non-empty finite subset $M_{2}$ of $A_{2}$ such that $B_{2}=\cup\left\{U: U \in M_{2}\right\}$. Now $M_{2}^{\prime}=\left\{U \cap Y: U \in M_{2}\right\} \subseteq A_{2}^{\prime}$ where $B_{2}^{\prime}=\cup\left\{V: V \in M_{2}^{\prime}\right\}$ is a legitimate move for TWO in $G\left(\mathcal{C}_{\mathcal{Y}}, \mathcal{I}_{\mathcal{Y}}\right)$ on $Y$. In $n-t h$ inning, Player ONE looks to the $n-t h$ move $A_{n}^{\prime}=\delta^{\prime}\left(A_{1}^{\prime}, B_{1}^{\prime}, \ldots, A_{n-1}^{\prime}, B_{n-1}^{\prime}\right)$ of player ONE in $G\left(\mathcal{C}_{\mathcal{Y}}, \mathcal{I}_{\mathcal{Y}}\right)$ on $Y$ and take $A_{n}=\delta\left(A_{1}, B_{1}, \ldots, A_{n-1}, B_{n-1}\right)=A_{n}^{\prime} \cup(X-Y)$ to be the $n-t h$ move in $G(\mathcal{C}, \mathcal{I})$ on $X$. TWO responds by selecting a non-empty finite subset 
$M_{n}$ of $A_{n}$ such that $B_{n}=\cup\left\{U: U \in M_{n}\right\}$. Now $M_{n}^{\prime}=\left\{U \cap Y: U \in M_{n}\right\} \subseteq A_{n}^{\prime}$ where $B_{n}^{\prime}=\cup\left\{V: V \in M_{n}^{\prime}\right\}$ is a legitimate move for TWO in $G\left(\mathcal{C}_{\mathcal{Y}}, \mathcal{I}_{\mathcal{Y}}\right)$ on $Y$. Now, since $\delta^{\prime}$ is a winning strategy for ONE in $G\left(\mathcal{C}_{\mathcal{Y}}, \mathcal{I}_{\mathcal{Y}}\right)$ on $Y$ implies $X-\cup\left\{B_{n}^{\prime}: n \in N\right\} \in I_{Y}$. So, $X-\cup\left\{B_{n}: n \in N\right\} \in I$. Hence, $\delta$ is a winning strategy for ONE in $G(\mathcal{C}, \mathcal{I})$ on $X$.

In similar way we can prove (ii) and (iii).

Theorem 2.11. If $f:(X, \tau) \rightarrow\left(Y, \tau^{\prime}\right)$ is a homeomorphism function, $I$ is an ideal on $X$ and $\mathcal{C}$ (resp. $\mathcal{C}^{\prime}$ ) a family of all open covers on $X$ (resp. $Y$ ), then the following statements are hold.

(i) $\mathrm{ONE} \uparrow G(\mathcal{C}, \mathcal{I})$ on $X$ if and only if $O N E \uparrow G\left(\mathcal{C}^{\prime},\{(\mathcal{I}))\right.$ on $Y$.

(ii) $O N E \uparrow G_{D}(\mathcal{C}, \mathcal{I})$ on $X$ if and only if $O N E \uparrow G_{D}\left(\mathcal{C}^{\prime},\{(\mathcal{I}))\right.$ on $Y$.

(iii) $O N E \uparrow G_{O}(\mathcal{C}, \mathcal{I})$ on $X$ if and only if $O N E \uparrow G_{O}\left(\mathcal{C}^{\prime},\{(\mathcal{I}))\right.$ on $Y$.

Proposition 2.12. If $(X, \tau, I)$ is an ideal topological space satisfying the Meneger property, then the following statements are hold.

(i) $T W O \downarrow G(\mathcal{C}, \mathcal{I})$.

(ii) $T W O \downarrow G_{D}(\mathcal{C}, \mathcal{I})$.

(iii) $T W O \downarrow G_{O}(\mathcal{C}, \mathcal{I})$.

Lemma 2.13. If $(X, \tau)$ is a topological space and $\tau-\{\varnothing\}$ has a finite intersection property, then the interior of every proper closed subset $F$ of $X$ is empty.

Proposition 2.14. If $\left(X, \tau, I_{n}\right)$ is an ideal topological space such that $\tau-\{\varnothing\}$ has a finite intersection property, then the following statements are hold.

(i) $T W O \uparrow G(\mathcal{C}, \mathcal{I})$.

(ii) $\mathrm{TWO} \uparrow G_{D}(\mathcal{C}, \mathcal{I})$.

(iii) $T W O \uparrow G_{O}(\mathcal{C}, \mathcal{I})$.

Proof. (i): Let $T$ be a strategy for player TWO in $G(C, I)$. In the $n-t h$ inning, ONE chooses $A_{n} \in \mathcal{C}$ and TWO responds by selecting a non-empty finite subset $M_{n}$ of $A_{n}$ such that $B_{n}=\cup\left\{U: U \in M_{n}\right\}$. By Lemma 2.13, $X-\cup\left\{B_{n}: n \in N\right\} \in I_{n}$. Hence, ONE wins the play $A_{1}, B_{1}, \ldots, A_{n}, B_{n}, \ldots$ and so TWO has no winning strategy.

The proof of (ii) and (iii) Follows from (i) and the hereditary property of an ideal.

Proposition 2.15. If $\left(X, \tau, I_{K}\right)$ is an ideal topological space such that $X$ is compact space, then the following statements are hold. 
(i) $T W O \uparrow G(\mathcal{C}, \mathcal{I})$

(ii) $T W O \uparrow G_{D}(\mathcal{C}, \mathcal{I})$.

(iii) $T W O \uparrow G_{O}(\mathcal{C}, \mathcal{I})$.

\section{Games and $\tau^{*}$-Open Covers}

In this section, we introduce new games by using some types of covering for ideal topological spaces.

Definition 3.1. Let $(X, \tau, I)$ be an ideal topological space and $\mathcal{C}$ (resp. $\left.\mathcal{C}^{*}\right)$ a collection of all open (resp. $\tau^{*}$-open) covers for $X$. We define the infinitely long game $G\left(\mathcal{C}^{*}, \mathcal{C}\right)$ as follows: They play an inning for each positive integer. In the $n$-th inning, ONE chooses $A_{n} \in \mathcal{C}^{*}$ and TWO responds by selecting a non-empty finite subset $M_{n}$ of $A_{n}$ such that $B_{n}=\cup\left\{U: U \in M_{n}\right\}$ is an open set. TWO wins the play $A_{1}, B_{1}, \ldots, A_{n}, B_{n}, \ldots$ of this game if $\left\{B_{n}: n \in N\right\}$ is an open cover for $X$. Otherwise, ONE wins.

Remark 3.2. Let $(X, \tau, I)$ be an ideal topological space. If $I=\{\varnothing\}$, then the two games Meneger $(X)$ and $G\left(\mathcal{C}^{*}, \mathcal{C}\right)$ are equivalent.

Proposition 3.3. Let $(X, \tau, I)$ be an ideal topological space. If TWO has a winning strategy in $G\left(\mathcal{C}^{*}, \mathcal{C}\right)$, then $O N E$ has a winning strategy in $G(\mathcal{C})$.

Proof. Let $\mathrm{T}$ be a winning strategy for TWO in $G\left(\mathcal{C}^{*}, \mathcal{C}\right)$. We define a strategy $\delta$ for ONE to be a winning strategy in $G(\mathcal{C})$. Let $A_{1}$ be the first move for ONE in $G\left(\mathcal{C}^{*}, \mathcal{C}\right)$. Player ONE in $G(\mathcal{C})$ looks to the first move for player TWO in $G\left(\mathcal{C}^{*}, \mathcal{C}\right)$, say $M_{1}$, which is finite subset of $A_{1}$ such that $B_{1}=T\left(A_{1}\right)=$ $\cup\left\{U: U \in M_{1}\right\}$ is an open subset of $X$. Then take any open cover $\mathcal{U}_{\infty}$ for $X$ and choose $A_{1}^{\prime}=\left\{B_{1} \cup V: V \in \mathcal{U}_{\infty}\right\}$ to be the first move for player ONE in $G(\mathcal{C}), \delta(\varnothing)=\mathcal{A}_{\infty}^{\prime}$. TWO responds by selecting a non-empty finite subset $M_{1}^{\prime}$ of $A_{1}^{\prime}$ such that $B_{1}^{\prime}=\cup\left\{V: V \in M_{1}^{\prime}\right\}$. Let $A_{2}$ be the second move for ONE in $G\left(\mathcal{C}^{*}, \mathcal{C}\right)$. Player ONE in $G(\mathcal{C})$ looks to the second move for player TWO in $G\left(\mathcal{C}^{*}, \mathcal{C}\right)$, say $M_{2}$ which is finite subset of $A_{2}$ such that $B_{2}=T\left(A_{1}, B_{1}, A_{2}\right)=$ $\cup\left\{U: U \in M_{2}\right\}$ is an open subset of $X$. Then take any open cover $\mathcal{U}_{\in}$ for $X$ and choose $A_{2}^{\prime}=\left\{B_{2} \cup V: V \in \mathcal{U}_{\in}\right\}$ to be the second move for player ONE in $G(\mathcal{C})$ such that $\delta\left(A_{1}^{\prime}, B_{1}^{\prime}\right)=A_{2}^{\prime}$. TWO responds by selecting a non-empty finite subset $M_{2}^{\prime}$ of $A_{2}^{\prime}$ such that $B_{2}^{\prime}=\cup\left\{V: V \in M_{2}^{\prime}\right\}$. In $n$ - th inning, let $A_{n}$ be the $n$-th move for ONE in $G\left(\mathcal{C}^{*}, \mathcal{C}\right)$. Player ONE in $G(\mathcal{C})$ looks to the $n-t h$ move for player TWO in $G\left(\mathcal{C}^{*}, \mathcal{C}\right)$, say $M_{n}$ which is finite subset of $A_{n}$ such that $B_{n}=T\left(A_{1}, B_{1}, \ldots, A_{n-1}, B_{n-1}, A_{n}\right)=\cup\left\{U: U \in M_{n}\right\}$ is an open subset of $X$. 
Then take any open cover $\mathcal{U}$ for $X$ and choose $A_{n}^{\prime}=\left\{B_{n} \cup V: V \in \mathcal{U}\right\}$ to be the $n$-th move for player ONE in $G(C)$ such that $\delta\left(A_{1}^{\prime}, B_{1}^{\prime}, \ldots, A_{n-1}^{\prime}, B_{n-1}^{\prime}\right)=A_{n}^{\prime}$. TWO responds by selecting a non-empty finite subset $M_{n}^{\prime}$ of $A_{n}^{\prime}$ such that $B_{n}^{\prime}=\cup\left\{V: V \in M_{n}^{\prime}\right\}$. Since $T$ is a winning strategy for TWO in $G\left(C^{*}, C\right)$, then $\left\{B_{n}: n \in N\right\}$ is an open cover for $X$. Implies, $X=\cup\left\{B_{n}^{\prime}: n \in N\right\}$. So $\delta$ is a winning strategy for ONE in $G(\mathcal{C})$.

Proposition 3.4. Let $(X, \tau, I)$ be an ideal topological space such that $I=\{\varnothing\}$ and $\mathcal{C}$ has a finite intersection property. If $O N E$ has a winning strategy in $G(\mathcal{C})$, then $T W O$ has a winning strategy in $G\left(\mathcal{C}^{*}, \mathcal{C}\right)$.

Proof. Let $\delta$ be a winning strategy for ONE in $G(\mathcal{C})$. We define a strategy $T$ for TWO to be a winning strategy in $G\left(\mathcal{C}^{*}, \mathcal{C}\right)$. Let $A_{1} \in \mathcal{C}^{*}$ be the first move for ONE in $G\left(\mathcal{C}^{*}, \mathcal{C}\right)$ and $A_{1}^{\prime}$ be the first move for ONE in $G(\mathcal{C})$ where $\delta(\varnothing)=A_{1}^{\prime}$. Since $I=\{\varnothing\}$ and $\mathcal{C}$ has a finite intersection property, then $A_{1} \cap A_{1}^{\prime}$ is nonempty. Player TWO in $G\left(\mathcal{C}^{*}, \mathcal{C}\right)$ choose $M_{1}$ to be a finite subset of $A_{1} \cap A_{1}^{\prime}$ such that $T\left(A_{1}\right)=B_{1}=\cup\left\{U: U \in M_{1}\right\}$ is an open subset of $X$ which is a legitimate move for TWO in $G(\mathcal{C})$. Let $A_{2} \in \mathcal{C}^{*}$ be the second move for ONE in $G\left(\mathcal{C}^{*}, \mathcal{C}\right)$ and $A_{2}^{\prime}$ be the second move for ONE in $G(\mathcal{C})$ where $\delta\left(A_{1}^{\prime}, B_{1}^{\prime}\right)=A_{2}^{\prime}$. Player TWO in $G\left(\mathcal{C}^{*}, \mathcal{C}\right)$ choose $M_{2}$ to be a finite subset of $A_{2} \cap A_{2}^{\prime}$ such that $T\left(A_{1}, B_{1}, A_{2}\right)=B_{2}=\cup\left\{U: U \in M_{2}\right\}$ is an open subset of $X$ which is a legitimate move for TWO in $G(\mathcal{C})$. In $n-t h$ inning. Let $A_{n} \in \mathcal{C}^{*}$ be the $n-t h$ move for ONE in $G\left(\mathcal{C}^{*}, \mathcal{C}\right)$ and $A_{n}^{\prime}$ be the $n-t h$ move for ONE in $G(\mathcal{C})$ where $\delta\left(A_{1}^{\prime}, B_{1}^{\prime}, \ldots, A_{n-1}^{\prime}, B_{n-1}^{\prime}\right)=A_{n}^{\prime}$. Player TWO in $G\left(\mathcal{C}^{*}, \mathcal{C}\right)$ choose $M_{n}$ to be a finite subset of $A_{n} \cap A_{n}^{\prime}$ such that $T\left(A_{1}, B_{1}, \ldots, A_{n-1}, B_{n-1}, A_{n}\right)=B_{n}=\cup\{U$ : $\left.U \in M_{n}\right\}$ is an open subset of $X$ which is a legitimate move for TWO in $G(\mathcal{C})$. Since $\delta$ is a winning strategy for ONE in $G(C)$, then $X=\cup\left\{B_{n}: n \in N\right\}$. Hence, $T$ is a winning strategy for TWO in $G\left(\mathcal{C}^{*}, \mathcal{C}\right)$.

Proposition 3.5. Let $(X, \tau, I)$ be an ideal topological space. If $I=\mathcal{P}(\mathcal{X})$ and $\tau \subset \mathcal{P}(\mathcal{X})$, then $O N E \uparrow G\left(\mathcal{C}^{*}, \mathcal{C}\right)$.

Proof. Since $\tau \subset \mathcal{P}(\mathcal{X})$, this means there exists $x_{0} \in X$ such that the only open set containing $x_{0}$ is $X$. So $I=\mathcal{P}(\mathcal{X})$, implies $\tau^{*}=\mathcal{P}(\mathcal{X})$. Now, in every $n$ - th inning, ONE must choose a $\tau^{*}$-open cover $A_{n}=\{\{x\}: x \in X\}$. Therefore, for every choosing non-empty finite subset $M_{n}$ of $A_{n}$ for player TWO $\left\{x_{0}\right\} \notin M_{n}$ such that $x_{0} \notin B_{n}=\cup\left\{U: U \in M_{n}\right\}$ is an open subset of $X$. Hence, $\left\{B_{n}: n \in N\right\} \notin \mathcal{C}$. So, ONE wins the play.

Proposition 3.6. If $\left(X, \tau, I_{f}\right)$ is an ideal indiscrete topological space, then $T W O \uparrow G\left(\mathcal{C}^{*}, \mathcal{C}\right)$. 
Proof. In $n$-th inning, for any choosing $A_{n}$ for ONE there are two probabilities. Either $A_{n} \in \mathcal{C}$ or $A_{n} \notin \mathcal{C}$. If $A_{n} \in \mathcal{C}$, then TWO can choose a finite subcover for $X$. If $A_{n} \notin \mathcal{C}$, then this implies there exists a $\tau^{*}$-open set $U \in A_{n}$ which it is complement is finite. Let $X-U=\left\{x_{1}, x_{2}, \ldots, x_{n}\right\}$. TWO must choose $M_{n}=\left\{U, U_{1}, U_{2}, \ldots, U_{n}\right\}$ such that $x_{i} \in U_{i}, i=1,2, \ldots, n$ and $B_{n}=\cup\{U$ : $\left.U \in M_{n}\right\}=X$. Hence, TWO wins the play.

Proposition 3.7. Let $\left(X, \tau_{c o}, I\right)$ be an ideal topological space, where $\tau_{c o}$ is the co-finite topology on $X$. If $I=\{\varnothing\}$, then $T W O \uparrow G\left(\mathcal{C}^{*}, \mathcal{C}\right)$.

Proposition 3.8. Let $f:(X, \tau, I) \rightarrow(Y, \sigma, f(I))$ be a bijective, open and *-continuous function. If $T W O \uparrow G\left(\mathcal{C}^{*}, \mathcal{C}\right)$ in $(X, \tau, I)$, then $T W O \uparrow G\left(\mathcal{C}^{*}, \mathcal{C}\right)$ in $(Y, \sigma, f(I))$.

\section{Games in I-Compact Spaces}

In this section, we use the concept of $I$-compact spaces to define new game say $G(X, I)$ and study the relationships between this game and the terms of selection principles.

Definition 4.1. Let $(X, \tau, I)$ be an ideal topological space. We define the infinitely long game $G(X, I)$ as follows: They play an inning for each positive integer. In the $n$-th inning, ONE chooses $I$-compact subset $H_{n}$ of $X$ and TWO responds by selecting an $I$-compact subset $M_{n}$ of $X$ such that $H_{n} \cap M_{n}=\varnothing$. ONE wins the play $H_{1}, M_{1}, \ldots, H_{n}, M_{n}, \ldots$ of this game if $\left\{M_{n}: n \in N\right\}$ is locally finite and $X-\cup\left\{M_{n}: n \in N\right\} \in I$. Otherwise, TWO wins.

Proposition 4.2. If $(X, \tau, I)$ is an $I$-compact space and $I=\mathcal{P}(\mathcal{X})$, then ONEץ $G(X, I)$.

Proposition 4.3. If $(X, \tau, I)$ is an $I$-compact space and $I \subset \mathcal{P}(\mathcal{X})$, then $O N E \downarrow G(X, I)$.

In the next Theorems, we can use the natural game $G_{1}(\mathcal{A}, \mathcal{B})$ to explain the win state of chances for both players in the game $G(X, I)$, where $\mathcal{A}$ and $\mathcal{B}$ are defined as follows:

Let $\mathcal{A}=\left\{\mathcal{A}_{\backslash}: \backslash \in *\right\}$ where $A_{n}$ is a family of $I$-compact subsets of $X$ satisfying that for each $I$-compact subsets $F$ of $X$ there exists $D \in A_{n}$ such that $F \cap D=\varnothing$.

If $X$ is non $I$-compact space then, for each $I$-compact subset $F$ of $X$ there exists $\{x\} \subseteq X-F$ such that $\{x\} \cap F=\varnothing$. 
Let $\mathcal{B}=\{\mathcal{B} \backslash: \backslash \in *\}$ where $B_{n}$ is an infinite locally finite family of $I$ compact subsets of $X$.

Theorem 4.4. Let $(X, \tau, I)$ be a non $I$-compact space such that $I=\mathcal{P}(\mathcal{X})$. Then $O N E \uparrow G(X, I)$ if and only if $T W O \uparrow G_{1}(\mathcal{A}, \mathcal{B})$.

Proof. Necessity, let $\delta$ be a winning strategy for ONE in $G(X, I)$. We define $T$ to be a winning strategy for TWO in $G_{1}(\mathcal{A}, \mathcal{B})$. In the first move of $G_{1}(\mathcal{A}, \mathcal{B})$, ONE choose $A_{1} \in A$. Player TWO in $G_{1}(\mathcal{A}, \mathcal{B})$ looks to the first move for player ONE in $G(X, I)$, say $H_{1}=\delta(\varnothing)$, and choose $T\left(A_{1}\right)=M_{1}$ such that $M_{1} \in A_{1}$ and $H_{1} \cap M_{1}=\varnothing$ which is possible by definition of $A . M_{1}$ is a legitimate move for TWO in $G(X, I)$. In the second move of $G_{1}(\mathcal{A}, \mathcal{B})$, ONE choose $A_{2} \in \mathcal{A}$. Player TWO in $G_{1}(\mathcal{A}, \mathcal{B})$ looks to the second move for player ONE in $G(X, I)$, say $H_{2}=\delta\left(H_{1}, M_{1}\right)$, and choose $T\left(A_{1}, M_{1}, A_{2}\right)=M_{2}$ such that $M_{2} \in A_{2}$ and $H_{2} \cap M_{2}=\varnothing . M_{2}$ is a legitimate move for TWO in $G(X, I)$. In the $n-t h$ inning of $G_{1}(\mathcal{A}, \mathcal{B})$, ONE choose $A_{n} \in \mathcal{A}$. Player TWO in $G_{1}(\mathcal{A}, \mathcal{B})$ looks to the $n-t h$ move for player ONE in $G(X, I)$, say $H_{n}=\delta\left(H_{1}, M_{1}, \ldots, H_{n-1}, M_{n-1}\right)$, and choose $T\left(A_{1}, M_{1}, \ldots, A_{n-1}, M_{n-1}, A_{n}\right)=M_{n}$ such that $M_{n} \in A_{n}$ and $H_{n} \cap M_{n}=\varnothing . M_{n}$ is a legitimate move for TWO in $G(X, I)$. Since $\delta$ is a winning strategy for ONE in $G(X, I)$, then $\left\{M_{1}, M_{2}, \ldots, M_{n}\right\}$ is locally finite family and $X-\cup\left\{M_{n}: n \in N\right\} \in I$. Hence, $\left\{M_{n}: n \in N\right\} \in \mathcal{B}$. So, $\mathrm{T}$ is a winning strategy for TWO in $G_{1}(\mathcal{A}, \mathcal{B})$.

Sufficient; let $W_{1}=\left\{K: K\right.$ is $I$-compact subset of $X$ such that $T\left(A_{1}\right) \neq K$ for each $\left.A_{1} \in \mathcal{A}\right\}$. We claim that $W_{1} \notin \mathcal{A}$. If $W_{1} \in \mathcal{A}$, then $W_{1}$ is a legitimate move for ONE in $G_{1}(\mathcal{A}, \mathcal{B})$. Consider that TWO responds by selecting $D=$ $T\left(W_{1}\right)$, implies $D \in W_{1}$. This is a contradiction of definition of $W_{1}$. Since $W_{1} \notin \mathcal{A}$, then there exists a non-empty $I$-compact subset $H_{1}$ of $X$ satisfying that for each $K \in W_{1}$ implies $K \cap H_{1} \neq \varnothing$. Now, in the first move of $G(X, I)$, ONE choose $H_{1}=\delta(\varnothing)$. TWO responds by selecting $M_{1}$ such that $H_{1} \cap M_{1}=$ $\varnothing$, implies $M_{1} \notin W_{1}$. Let $A_{1} \in \mathcal{A}$ be the first move of $\mathrm{ONE}$ in $G_{1}(\mathcal{A}, \mathcal{B})$ such that $M_{1}=T\left(A_{1}\right)$. In the second move, let $W_{2}=\{K: K$ is $I$-compact subset of $X$, such that $T\left(A_{1}, M_{1}, A_{2}\right) \neq K$ for each $\left.A_{2} \in \mathcal{A}\right\}$. Since $W_{2} \notin \mathcal{A}$, then there exists a non-empty $I$-compact subset $H_{2}$ of $X$ satisfying that for each $K \in W_{2}$ implies $K \cap H_{2} \neq \varnothing$. Now, in the second move of $G(X, I)$, ONE choose $H_{2}=\delta\left(H_{1}, M_{1}\right)$. TWO responds by selecting $M_{2}$ such that $H_{2} \cap M_{2}=\varnothing$, implies $M_{2} \notin W_{2}$. Let $A_{2} \in \mathcal{A}$ be the second move of ONE in $G_{1}(\mathcal{A}, \mathcal{B})$ such that $M_{2}=T\left(A_{1}, M_{1}\right)$. In $n-t h$ inning, let $W_{n}=\{K: K$ is $I$-compact subset of $X$ such that $T\left(A_{1}, M_{1}, \ldots, A_{n-1}, M_{n-1}, A_{n}\right) \neq K$ for each $\left.A_{n} \in \mathcal{A}\right\}$. Since $W_{n} \notin \mathcal{A}$, then there exists a non-empty $I$-compact subset $H_{n}$ of $X$ satisfying that for each $K \in W_{n}$ implies $K \cap H_{n} \neq \varnothing$. Now, in the $n-t h$ move of $G(X, I)$, 
ONE choose $H_{n}=\delta\left(H_{1}, M_{1}, \ldots, H_{n-1}, M_{n-1}\right)$. TWO responds by selecting $M_{n}$ such that $H_{n} \cap M_{n}=\varnothing$, implies $M_{n} \notin W_{n}$. Let $A_{n} \in \mathcal{A}$ be the $n-t h$ move of $\mathrm{ONE}$ in $G_{1}(\mathcal{A}, \mathcal{B})$ such that $M_{n}=T\left(A_{1}, M_{1}, \ldots, A_{n-1}, M_{n-1}, A_{n}\right)$. Since $T$ is winning strategy for TWO in $G_{1}(\mathcal{A}, \mathcal{B})$, then $\left\{M_{1}, M_{2}, \ldots, M_{n}\right\}$ is locally finite family. Hence, $\delta$ is a winning strategy for ONE in $G(X, I)$.

Theorem 4.5. For any non $I$-compact space $(X, \tau, I)$, TWO $G(X, I)$ if and only if $O N E \uparrow G_{1}(\mathcal{A}, \mathcal{B})$.

Proof. Necessity, let $T$ be a winning strategy for TWO in $G(X, I)$. We define $\delta$ to be a winning strategy for ONE in $G_{1}(\mathcal{A}, \mathcal{B})$. Let $H_{1}$ be the first move of ONE in $G(X, I)$ and $A_{1}=\left\{K: K\right.$ is $I$-compact subset of $X$ such that $\left.T\left(H_{1}\right)=K\right\}$. Implies, $A_{1} \in \mathcal{A}$ and $A_{1}$ is a legitimate move to be the first selecting of ONE in $G_{1}(\mathcal{A}, \mathcal{B})$. TWO, in $G_{1}(\mathcal{A}, \mathcal{B})$, responds by selecting $M_{1}=\delta\left(A_{1}\right)$ since $M_{1} \in A_{1}$, then $H_{1} \cap M_{1}=\varnothing$ and $T\left(H_{1}\right)=M_{1}$ is the first move for TWO in $G(X, I)$. In the second move, let $H_{2}$ be the second move of ONE in $G(X, I)$ and $A_{2}=\left\{K: K\right.$ is $I$-compact subset of $X$ such that $\left.T\left(H_{1}, M_{1}, H_{2}\right)=K\right\}$. Implies, $A_{2} \in \mathcal{A}$ and $A_{2}$ is a legitimate move to be the second selecting of ONE in $G_{1}(\mathcal{A}, \mathcal{B})$. TWO, in $G_{1}(\mathcal{A}, \mathcal{B})$, responds by selecting $M_{2}=\delta\left(A_{1}, M_{1}, A_{2}\right)$ since $M_{2} \in A_{2}$, then $T\left(H_{1}, M_{1}, H_{2}\right)=M_{2}$ and $H_{2} \cap M_{2}=\varnothing$. Now, in the $n$-th move of $G(X, I)$, ONE choose $H_{n}$. Let $A_{n}=\{K: K$ is $I$-compact subset of $X$ such that $\left.T\left(H_{1}, M_{1}, \ldots, H_{n-1}, M_{n-1}, H_{n}\right)=K\right\}$. Implies, $A_{n} \in \mathcal{A}$ and $A_{n}$ is a legitimate move to be the $n-t h$ selecting of ONE in $G_{1}(\mathcal{A}, \mathcal{B})$. TWO, in $G_{1}(\mathcal{A}, \mathcal{B})$, responds by selecting $M_{n}=\delta\left(A_{1}, M_{1}, \ldots, A_{n-1}, M_{n-1}, A_{n}\right)$ since $M_{n} \in A_{n}$, then $T\left(H_{1}, M_{1}, \ldots, H_{n-1}, M_{n-1}, H_{n}\right)=M_{n}$ and $H_{n} \cap M_{n}=\varnothing$. Since $T$ is a winning strategy for TWO in $G(X, I)$, then $\left\{M_{n}: n \in N\right\}$ is not locally finite family. Therefore, $\left\{M_{n}: n \in N\right\} \notin \mathcal{B}$. Hence, $\delta$ is a winning strategy for ONE in $G_{1}(\mathcal{A}, \mathcal{B})$.

Sufficient; let $\delta$ be a winning strategy for ONE in $G_{1}(\mathcal{A}, \mathcal{B})$. We define $T$ to be a winning strategy for TWO in $G(X, I)$. Player ONE in $G(X, I)$ choose an $I$-compact subset $H_{1}$ of $X$. TWO in $G(X, I)$ looks to the first move for player ONE in $G_{1}(\mathcal{A}, \mathcal{B})$, say $A_{1}=\delta(\varnothing)$, and choose $M_{1} \in A_{1}$ such that $H_{1} \cap M_{1}=\varnothing$ which is possible by definition of $\mathcal{A}, \mathcal{T}\left(\mathcal{H}_{\infty}\right)=\mathcal{M}_{\infty}$ which is a legitimate to be the first move for TWO in $G_{1}(\mathcal{A}, \mathcal{B})$. In the second move, Player ONE in $G(X, I)$ choose an $I$-compact subset $H_{2}$ of $X$. TWO in $G(X, I)$ looks to the second move for player ONE in $G_{1}(\mathcal{A}, \mathcal{B})$, say $A_{2}=\delta\left(A_{1}, M_{1}\right)$ and choose $M_{2} \in$ $A_{2}$ such that $H_{2} \cap M_{2}=\varnothing$ and $T\left(H_{1}, M_{1}, H_{2}\right)=M_{2}$ is a legitimate to be the second move for TWO in $G_{1}(\mathcal{A}, \mathcal{B})$. In the $n$-thinning, Player ONE in $G(X, I)$ choose an $I$-compact subset $H_{n}$ of $X$. TWO in $G(X, I)$ looks to the $n$-th move for player $\mathrm{ONE}$ in $G_{1}(\mathcal{A}, \mathcal{B})$, say $A_{n}=\delta\left(A_{1}, M_{1}, \ldots, A_{n-1}, M_{n-1}\right)$ and choose 
$M_{n} \in A_{n}$ such that $H_{n} \cap M_{n}=\varnothing$ and $T\left(H_{1}, M_{1}, \ldots, H_{n-1}, M_{n-1}, H_{n}\right)=M_{n}$ is a legitimate to be the $n-t h$ move for TWO in $G_{1}(\mathcal{A}, \mathcal{B})$. Since $\delta$ is a winning strategy for ONE in $G_{1}(\mathcal{A}, \mathcal{B})$, then $\left\{M_{n}: n \in N\right\}$ is not locally finite family. Hence, $\delta$ is a winning strategy for TWO in $G(X, I)$.

Theorem 4.6. For any two equivalent ideal topological spaces $(X, \tau, I)$ and $(Y, \sigma, f(I))$, the following statements are equivalent.

(i) $O N E \uparrow G(X, I)$.

(ii) $O N E \uparrow G(Y, f(I))$.

Proof. (i) $\Rightarrow$ (ii): Let $\delta$ be a winning strategy for ONE in $G(X, I)$. We will define $\delta^{\prime}$ to be a winning strategy for ONE in $G(Y, f(I))$. In the first move in $G(Y, f(I))$, ONE looks to the first move for player ONE in $G(X, I)$, say $H_{1}=\delta(\varnothing)$ and choose $f\left(H_{1}\right)$ to be the first move for player ONE in $G(Y, f(I))$ which is possible since $f\left(H_{1}\right)$ is an $f(I)$-compact subset of $Y$. TWO responds by selecting $f(I)$-compact subset $M_{1}$ of $Y$ such that $f\left(H_{1}\right) \cap M_{1}=\varnothing$. It is clear that $f^{-1}\left(M_{1}\right)$ is a legitimate to be the first move for TWO in $G(X, I)$. In the $n-t h$ inning in $G(Y, f(I))$ ONE looks to the $n-t h$ move for player ONE in $G(X, I)$, say $H_{n}=\delta\left(H_{1}, f^{-1}\left(M_{1}\right), \ldots, H_{n-1}, f^{-1}\left(M_{n-1}\right)\right)$ and choose $f\left(H_{n}\right)$ to be the $n$-th move for player ONE in $G(Y, f(I))$ which is possible since $f\left(H_{n}\right)$ is an $f(I)$-compact subset of $Y$. TWO responds by selecting $f(I)$-compact subset $M_{n}$ of $Y$ such that $f\left(H_{n}\right) \cap M_{n}=\varnothing$. It is clear that $f^{-1}\left(M_{n}\right)$ is a legitimate $n-t h$ move for TWO in $G(X, I)$. Since $\delta$ is a winning strategy for ONE in $G(X, I),\left\{f^{-1}\left(M_{n}\right): n \in N\right\}$ is locally compact and $X-\cup\left\{f^{-1}\left(M_{n}\right): n \in N\right\} \in$ I. Implies, $\left\{M_{n}: n \in N\right\}$ is locally compact and $X-\cup\left\{M_{n}: n \in N\right\} \in f(I)$. Hence, $\delta^{\prime}$ is a winning strategy for ONE in $G(Y, f(I))$.

In Similar way we can prove (ii) $\Rightarrow$ (i).

\section{References}

[1] I. T. Adamson, A general topology workbook, Printed on Acid- free Paper, Birkhauser, Boston, 1966.

[2] A.V. Arkhangel'skii, V.I. Ponomarev, Fundamentals of general topology-problems and exercises, Hindustan Pub. Corporation,Delhi, 1966.

[3] L. Babinkostova, Lj.D. R. Kocinac and M. Scheepers, Notes on selection principles in topology (I): Paracompactness, J. Korean Math. Soc. 42 (2005), No. 4, pp. 709-721.

[4] A. K. Chandra and L.J. Stockmeyer, Alternation, Proceedings, IEEE 17th Annual Symposium of Foundations of Computer Science (1976), 98-108.

[5] A. K. Chandra, D. Kozen and L.J. Stockmeyer, Alternation, Report RC 7489, IBM research division, Yorktown Heights, N.Y (1978). 
[6] J. Dontchev, M. Ganster and D. Rose, Ideal resolvability. Topology and its Appl. 93(1991), 1-16.

[7] D.H. Fremlin, A.W. Miller, On some properties of Hurewicz, Menger, and Rothberger, Fund. Math., 129 (1988), pp. 17-33.

[8] T.R. Hamlett and D. Rose, *-Topological properties, Internat. J. Math. and Math. Sci. Vol.13 No.3(1990),507-512.

[9] D. Jankovic and T. R. Hamlett, New topologies from old via ideals. Amer. Math. Monthly, 97(1990), 295- 310.

[10] K. Kuratowski, Topology, Vol I. New York: Academic Press, (1933).

[11] D. V. Rancin, Compactness modulo an ideal, Soviet Math. Dokl., 13(1)(1972), 131- 147.

[12] M. Scheepers, Combinatorics of open covers (I): Ramsey Theory, Topology and its Applications, Vol.69, Issue1, 14 March 1996, 31-62.

[13] V. Vaidyanathaswamy, The localization theory in set topology, Proc. Indian Acad. Sci. 20(1945), 51- 61. 